\title{
Effects of gain-scheduling methods in a classical wind turbine controller on wind turbine aeroservoelastic modes and loads
}

\author{
Tibaldi, Carlo; Henriksen, Lars Christian; Hansen, Morten Hartvig; Bak, Christian
}

\section{Published in:}

Proceedings of 32nd ASME Wind Energy Symposium

Link to article, DOI:

$10.2514 / 6.2014-0873$

Publication date:

2014

Document Version

Publisher's PDF, also known as Version of record

Link back to DTU Orbit

Citation (APA):

Tibaldi, C., Henriksen, L. C., Hansen, M. H., \& Bak, C. (2014). Effects of gain-scheduling methods in a classical wind turbine controller on wind turbine aeroservoelastic modes and loads. In Proceedings of 32nd ASME Wind Energy Symposium American Society of Mechanical Engineers. https://doi.org/10.2514/6.2014-0873

\section{General rights}

Copyright and moral rights for the publications made accessible in the public portal are retained by the authors and/or other copyright owners and it is a condition of accessing publications that users recognise and abide by the legal requirements associated with these rights.

- Users may download and print one copy of any publication from the public portal for the purpose of private study or research.

- You may not further distribute the material or use it for any profit-making activity or commercial gain

- You may freely distribute the URL identifying the publication in the public portal 


\title{
Effects of gain-scheduling methods in a classical wind turbine controller on wind turbine aeroservoelastic modes and loads
}

\author{
Tibaldi C, Henriksen LC, Hansen MH and Bak C \\ Department of Wind Energy, Technical University of Denmark
}

\begin{abstract}
The effects of different gain-scheduling methods for a classical wind turbine controller, operating in full load region, on the wind turbine aeroservoelastic modes and loads are investigated in this work. The different techniques are derived looking at the physical problem to take into account the changes in the aerodynamic characteristics as a function of the wind speed. The modal analysis is performed with a high-order linear aeroservoelastic model computed with the frequency based stability tool HAWCStab2. The time series of the wind turbines loads are computed with the non-linear time domain tool HAWC2. Results show changes in the natural frequency and in the damping ratio of the speed regulator mode and of the tower longitudinal mode when using the different gain-scheduling schemes.
\end{abstract}

\section{Introduction}

In this paper a comparison of different gain-scheduling techniques for a classical Proportional Integral (PI) collective pitch wind turbine controller is performed. In the above-rated region, where the PI controller is active to set the blades pitch angle, the aerodynamic characteristics of wind turbines are subject to significant variations. These differences are due to the changing wind speed, mean blade pitch angle, and blade deformation. Hence, a gain-scheduling technique that can compensate and take into account for these changes is needed to obtain uniform performances of the speed regulator.

Gain-scheduling techniques have been presented by different authors. These approaches differ mainly on the variable used for the parametrization and on the methodology to compute the coefficients of the gainscheduling function. $\varnothing \mathrm{ye}^{1}$ presented a method to account for the different sensitivity of the aerodynamic torque to pitch angle variations occurring at different wind speeds. In the method suggested by Øye the gains are changed linearly with the pitch angle. The gains of the PI controller and the coefficients used for the gainscheduling are derived with the pole placement of the rigid body drivetrain mode. This approach has also been used by Wright. ${ }^{2}$ Bossanyi $^{3}$ also suggests a linear gain-scheduling with respect to the pitch angle. In his works he also states that, since the thrust sensitivity varies in a different way, a different parametrization may be required to ensure good performances at all wind speeds. A previous investigation by Hansen ${ }^{4}$ has shown that the linear interpolation suggested by Øye does not guarantee the correct placement of the speed regulator mode for all the operational range. For high wind speeds the gain-scheduling method is not able to ensure the location of the pole and the value of the frequency increases for increasing wind speeds. Furthermore, the damping of the resultant regulator mode differs from the value set with the placement technique. Therefore, the need of a better gain-scheduling to guarantee a better location of the pole in all the operational range exists.

The purpose of this work is to characterize the performances of some gain-scheduling techniques. To achieve this a high-order linear aeroservoelastic model of a wind turbine is used to perform closed-loop modal analysis. The analysis is performed to take into account the possible coupling between the controller and the structure as shown in previous works. ${ }^{4,5}$ In this investigation the method suggested by $\varnothing y e^{1}$ is considered $^{2}$ as a baseline. This method is then extended as suggested by Hansen ${ }^{4}$ with a quadratic parametrization of the derivative of the aerodynamic torque with respect to the pitch angle. Finally, a modified gain-scheduling

*tlbl@dtu.dk 
is introduced to compensate the changes in the derivative of the aerodynamic torque with respect to the rotational rotor speed. The performances of the gain-scheduling techniques are evaluated by looking at the frequency and damping of the speed regulator mode and at relevant wind turbines loads. The investigation is performed using HAWCStab2 $2^{8,9,10}$ to generate a high-order linear aeroservoelastic model of the wind turbine and to perform the closed-loop modal analysis. HAWC2 ${ }^{6}$ is exploited to validate with a non-linear multi-body aeroservoelastic model the results obtained with HAWCStab2 and to identify how loads and performances are affected. The $5 \mathrm{MW}$ NREL reference turbine ${ }^{7}$ is used in the investigation. The new gainscheduling method shows an improvement in the positioning of the speed regulator mode, especially at high wind speeds. The frequency of the mode decreases getting closer to the intended value. Hence, higher rotor speed excursions and lower tower loads occur at high wind speeds compared to the traditional approach.

This paper is structured as follows. In the first part of this investigation three gain-scheduling are derived based on the same simplified model. The methods are then used with a single degree of freedom wind turbine model to evaluate the position of the speed regulator mode. Rotor speed responses to wind steps of the single degree of freedom linear model are compared with those obtained with HAWC2 with a fully stiff turbine. The gain-scheduling methods are then compared closing the loop with a high-order model. The analysis is performed comparing frequencies and damping of the different aeroservoelastic wind turbine modes. A comparison with HAWC2 is also performed with this model. Finally, the gain-scheduling are compared looking at their influence on some wind turbine loads.

\section{Linear aeroservoelastic wind turbine model}

The linearized model, used for the investigation is obtained with the in-house developed tool HAWCStab2. HAWCStab2 is an improved version of HAWCStab ${ }^{8}$ and it includes a different kinematics. The model is based on an analytical linearization of a non-linear finite beam element model coupled with an unsteady blade element momentum model of the blade aerodynamic. The aerodynamic model includes shed vorticity and dynamic stall. The model does not include dynamic inflow and assumes frozen wake. A detailed description of the model is provided by Hansen. ${ }^{9}$ An extensive validation and analysis of the open-loop performances of the tool are provided by Sønderby and Hansen. ${ }^{10}$ The controller used to close the loop is a simplified linearization of the controller described by Hansen and Henriksen. ${ }^{11}$ The controller included in HAWCStab2 regards only the above-rated region. In this region the primary controller objectives are to maintain constant rotational speed and constant power or torque. The linearized controller includes the PI pitch controller, a second-order filter of the rotor speed feedback and a first-order filter of the measured pitch angle. The filtered pitch angle is required by the gain-scheduling. The linearized controller is described in detail by Hansen. ${ }^{4}$ The closed-loop high fidelity model used for the investigation is composed by 834 dynamic states, 336 aerodynamic and 498 structural.

\section{Controller tuning and gain-scheduling techniques}

In this section the method to perform the controller tuning is shown and the different gain-scheduling schemes are introduced.

The tuning of the proportional and integral gains $\left(k_{P}\right.$ and $\left.k_{I}\right)$ of the PI controller is based on pole placement of the rigid body drivetrain mode introduced by Øye. ${ }^{1}$ The method assumes rigid turbine, quasisteady aerodynamics, no rotor speed filters, and no pitch actuators. Under these assumptions the open-loop system can be described with a single degree of freedom second-order system

$$
I \dot{\Omega}=Q(V, \Omega, \theta)-Q_{g}(\Omega)
$$

where $\dot{\Omega}$ is the first time derivative of the rotational speed, $I$ is the total drivetrain inertia including rotor, shaft, gearbox and generator, $Q_{g}$ is the controlled generator torque that for the given control law depends on the rotor speed, and $Q$ is the aerodynamic torque that depends on the wind speed $V$, the rotor speed, and the pitch angle $\theta$. After linearizing Eq. (1) around the operational steady states, the second-order system can be written as

$$
I \ddot{\phi}+\left(\frac{\partial Q_{g}}{\partial \Omega}-\frac{\partial Q}{\partial \Omega}\right) \dot{\phi}-\frac{\partial Q}{\partial \theta} \delta \theta=0
$$

where $\dot{\phi}$ is the rotor speed variation relative to the rated rotor speed $\dot{\phi}=\Omega-\Omega_{R}, \delta \theta$ is the pitch angle variation, $\frac{\partial Q_{g}}{\partial \Omega}$ is the partial derivative of the generator torque with respect to the rotor speed and $\frac{\partial Q}{\partial \Omega}$ and 
$\frac{\partial Q}{\partial \theta}$ are the partial derivatives of the aerodynamic torque with respect to the rotor speed and the pitch angle respectively. The last two terms, $\frac{\partial Q}{\partial \Omega}$ and $\frac{\partial Q}{\partial \theta}$, can also be referred to as aerodynamic damping and aerodynamic gain respectively. The dependency on the wind speed of the aerodynamic damping has been neglected since the focus is on the homogeneous system to perform eigenvalue analysis. The linearized equations of the PI pitch controller are

$$
\delta \theta=k_{P} \dot{\phi}+k_{I} \phi
$$

where $\phi$ is the integral of the of the rotor speed variation $\dot{\phi}$. If the controller is included in the system the resulting closed-loop equation is

$$
I \ddot{\phi}+\left(\frac{\partial Q_{g}}{\partial \Omega}-\frac{\partial Q}{\partial \Omega}-k_{P} \frac{\partial Q}{\partial \theta}\right) \dot{\phi}-k_{I} \frac{\partial Q}{\partial \theta} \phi=0 .
$$

The pole placement is performed imposing the natural frequency $\omega_{\Omega}$ and damping ratio $\xi_{\Omega}$ to the secondorder system in Eq. (4).

$$
\omega_{\Omega}^{2}=-\frac{k_{I}}{I} \frac{\partial Q}{\partial \theta} \quad \xi_{\Omega}=-\frac{\frac{\partial Q_{g}}{\partial \Omega}-\frac{\partial Q}{\partial \Omega}-k_{P} \frac{\partial Q}{\partial \theta}}{2 k_{I} \frac{\partial Q}{\partial \theta}}
$$

The two equations in Eq. (5) can be solved for the two PI gains

$$
k_{P}=-\frac{2 \xi_{\Omega} \omega_{\Omega} I-\frac{\partial Q_{g}}{\partial \Omega}+\frac{\partial Q}{\partial \Omega}}{\frac{\partial Q}{\partial \theta}} \quad k_{I}=-\frac{\omega_{\Omega}^{2} I}{\frac{\partial Q}{\partial \theta}}
$$

The gains obtained with this approach depend only on the frequency and damping of the speed regulator mode, the drivetrain inertia, the derivative of the generator torque with respect to the rotational speed, and the gradient of the aerodynamic torque. The frequency and the damping ratio of the speed regulator mode are usually selected to be lower than the first longitudinal tower mode frequency to avoid resonance conditions but high enough to avoid large rotor speed excursions. The derivative of the generator torque with respect to the rotational speed depends whether the wind turbine is regulated for constant torque ( $\frac{\partial Q_{g}}{\partial \Omega}=0$ ) or constant power ( $\frac{\partial Q_{g}}{\partial \Omega}=\frac{P_{r}}{\Omega^{2}}$ where $P_{r}$ is the rated power). In this work constant torque is selected, therefore $\frac{\partial Q_{g}}{\partial \Omega}$ is set to zero. The rotor inertia, for a fixed rotor, depends only on the changes in rotor diameter due to steady static blade deformations. For the $5 \mathrm{MW}$ NREL reference turbine the maximum variation of the inertia is $0.1 \%$. For this reason the drivetrain inertia can be approximated as constant. Both the aerodynamic gain and the aerodynamic damping depend on the operational conditions, hence the necessity to have a gain-scheduling scheme.

In this work the aerodynamic gains and damping are approximated with a polynomial approximation as functions of the pitch angle $\Theta$. The value of the pitch angle used for the gain-scheduling is obtain filtering with a first-order filter the measured pitch angle. In this investigation the time constant of the first-order filter is set to a high value to neglect its dynamic. If a quadratic fit is selected, the approximations are

$$
\left.\frac{\partial Q}{\partial \theta} \approx \frac{\partial Q}{\partial \theta}\right|_{0}\left(1+\frac{\Theta}{K_{1}}+\frac{\Theta^{2}}{K_{2}}\right)=\left.\left.\frac{\partial Q}{\partial \theta}\right|_{0} \frac{1}{\eta_{K}} \quad \frac{\partial Q}{\partial \Omega} \approx \frac{\partial Q}{\partial \Omega}\right|_{0}\left(1+\frac{\Theta}{K_{1, \Omega}}+\frac{\Theta^{2}}{K_{2, \Omega}}\right)=\left.\frac{\partial Q}{\partial \Omega}\right|_{0} \eta_{K, \Omega}
$$

where the parameters $K_{1}, K_{2}, K_{1, \Omega}$ and $K_{2, \Omega}$ can be computed from fitting of quasi-steady calculations. In this investigation the polynomials are fitted to the gradient computed with HAWCStab2 using a least-squares method. In the work by $\varnothing y \mathrm{y}^{1}$ and Hansen ${ }^{9}$ the influence of the aerodynamic damping is neglected for the tuning while the aerodynamic gain is approximated with a linear and quadratic fit respectively. Figures 1 and 2 on the following page show the aerodynamic gain and the aerodynamic damping. Values computed with HAWCStab2 and a linear and quadratic polynomial fitting are plotted. The figures show how the fitting is improved when using a quadratic approximation compared to the linear.

Substituting the approximations in Eq. (7) into the gains in Eq. (6) the gains can be written as

$$
k_{P}=k_{P, 0} \eta_{K}+k_{P, 0, \Omega} \eta_{K} \eta_{K, \Omega} \quad k_{I}=k_{I, 0} \eta_{K}
$$




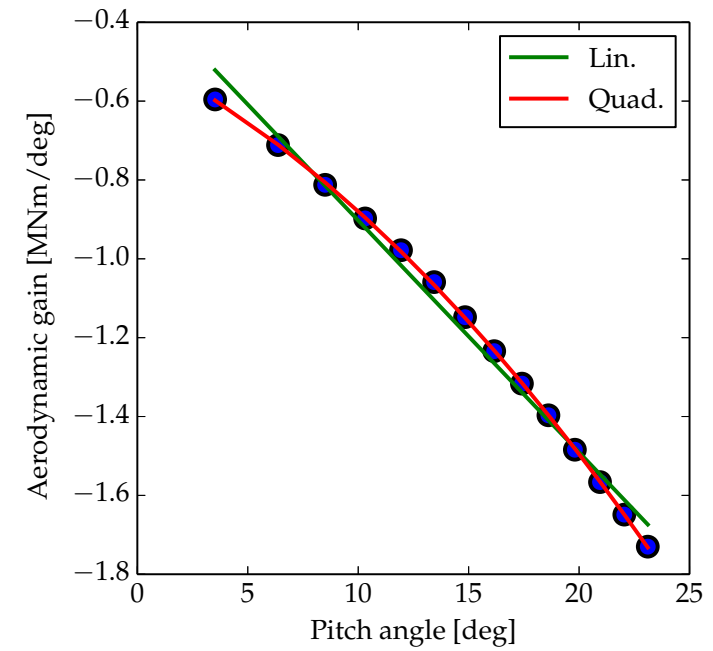

Figure 1. Partial derivative of the aerodynamic torque with respect to the pitch angle $\frac{\partial Q}{\partial \theta}$. Circles: HAWCStab2 computations. Curves: linear and quadratic least-squares fit.

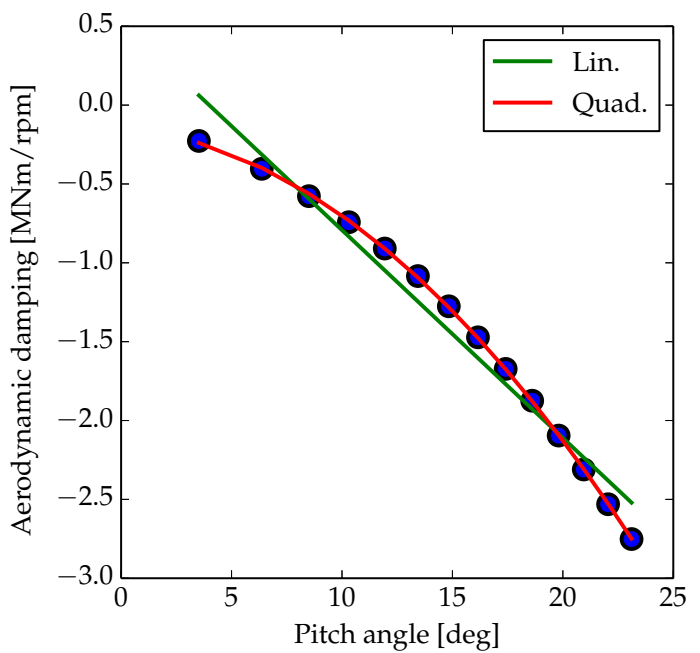

Figure 2. Partial derivative of the aerodynamic torque with respect to the rotor speed $\frac{\partial Q}{\partial \Omega}$. Circles: HAWCStab2 computations. Curves: linear and quadratic least-squares fit.

where

$$
k_{P, 0}=-\frac{2 \xi_{\Omega} \omega_{\Omega} I-\frac{\partial Q_{g}}{\partial \Omega}}{\left.\frac{\partial Q}{\partial \theta}\right|_{0}}, \quad k_{P, 0, \Omega}=-\frac{\left.\frac{\partial Q}{\partial \Omega}\right|_{0}}{\left.\frac{\partial Q}{\partial \theta}\right|_{0}} \quad \text { and } \quad k_{I, 0}=-\frac{\omega_{\Omega}^{2} I}{\frac{\partial Q}{\partial \theta}}
$$

Depending on whether a linear or quadratic polynomial approximation is selected and whether the aerodynamic damping is neglected, different gain-scheduling solutions can be obtained.

\section{A. PI gains}

In this work three different gain-scheduling schemes are compared:

- Lin. assumes a linear variation of the aerodynamic gain and no aerodynamic damping,

- Quad. assumes a quadratic variation of the aerodynamic gain and no aerodynamic damping,

- Quad.+Damp assumes a quadratic variation of the aerodynamic gain and damping.

Due to the different gain-scheduling the PI gains will differ from each other at each wind speed. Figure 3 on the next page shows the proportional and integral gains computed with the different gain-scheduling methods. A natural frequency $f_{n, \Omega}$ of $0.1 \mathrm{~Hz}$ and damping ratio $\xi_{\Omega}$ of 0.7 are assigned to the rotor speed regulator mode. A reference value is also shown where no gain-scheduling is used but the gains are computed using for each operational point the actual values of the gradient computed with HAWCStab2. This comparison allows evaluating how the different approaches perform compared to ideal reference values. Using a quadratic instead of a linear approximation for the aerodynamic gain does not significantly affect the controller gains. The larger difference is noticed at low pitch angles, at the beginning of the above-rated region. Here the linear fitting leads to higher proportional and integral gains compared to the reference values and those obtained with a quadratic fitting. Neglecting the aerodynamic damping only affects the proportional gain, as seen in Eq. (8). The differences can be noticed for increasing pitch angles. Without the aerodynamic damping the proportional gain results to be even more than twice the reference value. This first comparison shows that the linear approximation of the aerodynamic gain has poor agreement for low pitch angles with the reference values. Neglecting the aerodynamic damping $\frac{\partial Q}{\partial \Omega}$ penalizes the gains at higher wind speeds. The proportional gain is the most affected by the choice of gain-scheduling, consequently different performances are expected especially for the damping of the regulated mode. 


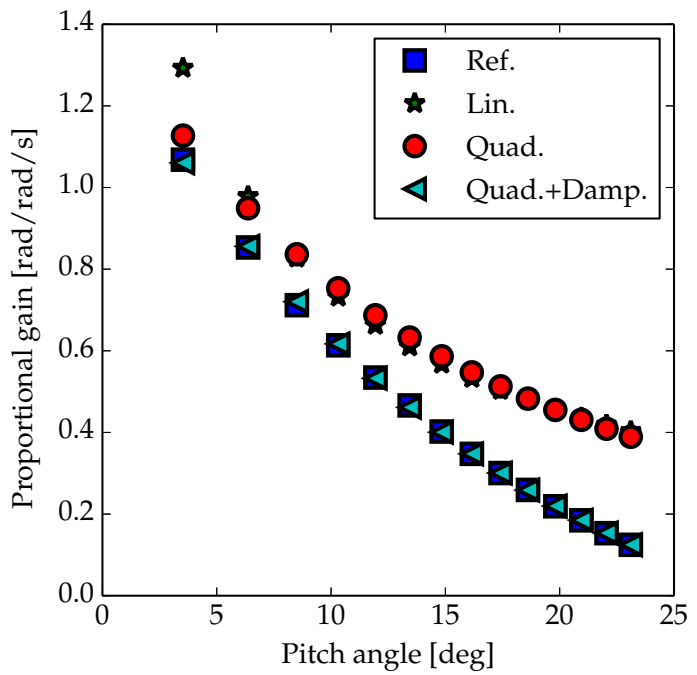

a) Proportional gain.

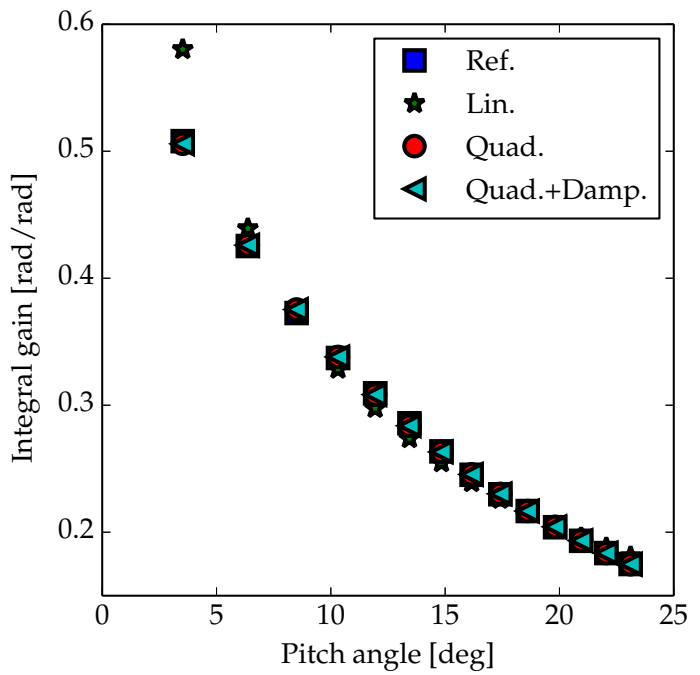

b) Integral gain.

Figure 3. Comparison of the proportional and integral gains computed with the different gain-scheduling methods. The reference gains are obtained without gain-scheduling and using the actual values of the gradient of the aerodynamic torque.

\section{Results}

In this section results from the closed-loop analysis are shown. First the investigation is carried out on a single degree of freedom model and then on a high-order model.

\section{A. Single degree of freedom}

In this section the three gain-scheduling methods are compared closing the loop with a single degree of freedom model of a wind turbine. The model used is the same as the one used for the tuning, hence the pole placement depends only on the gain-scheduling and it is not affected by the influence of other modes. Figure 4 on the following page shows the natural frequency and the damping ratio of the rotor speed regulator mode. The results obtained with the three gain-scheduling methods are compared with those obtained with the reference gains. The reference gains place the regulator mode exactly where it is desired to, $f_{n}=0.1 \mathrm{~Hz}$ and $\xi=0.7$. Even when using a single degree of freedom model significant differences can be noticed between the results obtained with the different techniques. If a quadratic fitting of the aerodynamic gain is selected an improvement in the frequency location can be noticed, Figure 4 on the next page a). The linear fitting generates a frequency that is almost $7 \%$ higher than the reference one at low pitch angles. With the quadratic fitting of the aerodynamic gain the natural frequency has a maximum discrepancy compare to the reference value of less than $0.5 \%$. Different results appear for the damping ratio in Figure $4 b$ ). If the aerodynamic damping is neglected in the gain-scheduling the damping ratio of the regulator mode differs from the placed value. The mode damping increases with the pitch angle and becomes over-damped for a pitch angle of $17 \mathrm{deg}$. When including the aerodynamic damping the resultant damping ratio is almost overlapped to the reference value. Figure 5 on the following page shows the trajectory of the regulator mode poles as function of the wind speed when a linear fitting of the aerodynamic gain is used and no aerodynamic damping is considered for the gain-scheduling. At low wind speeds the poles are complex-conjugate, hence the mode is undamped. When the wind speed increases the poles approach the real axis. Between $20 \mathrm{~m} / \mathrm{s}$ and $21 \mathrm{~m} / \mathrm{s}$ the poles become real and therefore the mode becomes over-damped. The distance between the poles keeps increasing for increasing wind speed leading to a further increase in the damping of the mode.

\section{Comparison of the linear and non-linear model}

The results obtained with the single degree of freedom model are here compared with HAWC2 simulations. The comparison is performed looking at the time response of the rotational speed to a step in the wind 


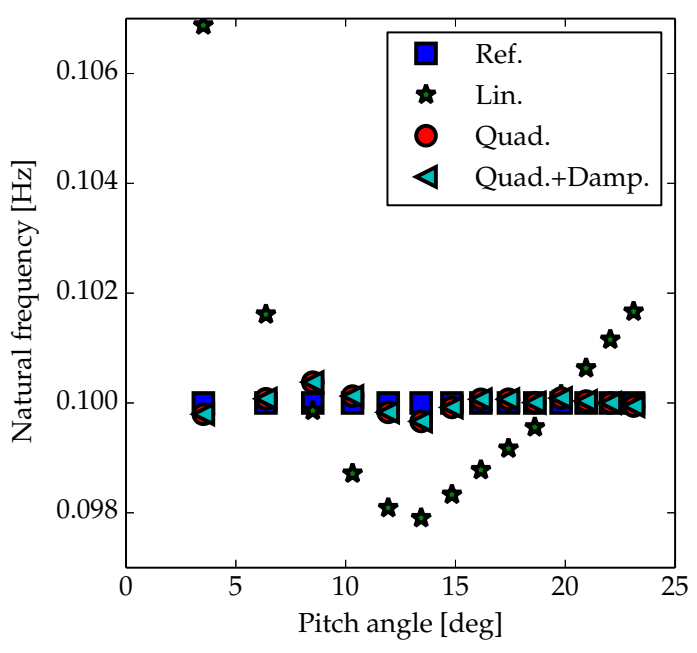

a) Natural frequency.

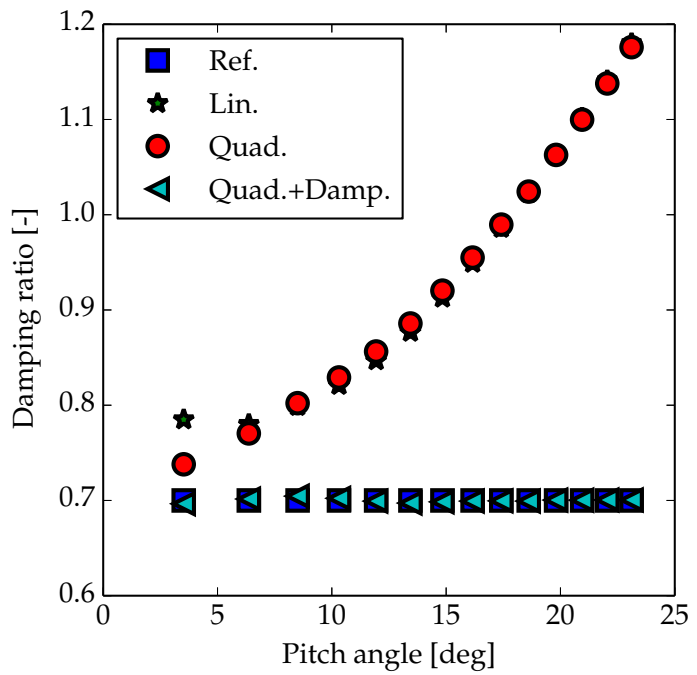

b) Damping ratio.

Figure 4. Natural frequency and the damping ratio of the rotor speed regulator mode using a single degree of freedom model of the wind turbine.

speed. The comparison aims at identifying if the two different codes agree on the location of the regulator mode. Here the aim is not to investigate the limit of the linear approximation with respect to the non-linear model. To have the same model assumptions as in the linear model, the structure in the HAWC2 model is made stiff and the dynamic stall model is disabled. Here the linear model is obtained with HAWCStab2. The wind speed step is of $0.5 \mathrm{~m} / \mathrm{s}$. For the HAWC2 simulations the wind step reaches the declared wind speed, e.g. the wind step at $15 \mathrm{~m} / \mathrm{s}$ goes from 14.5 to $15 \mathrm{~m} / \mathrm{s}$. For the HAWCStab2 responses the linear model at the final wind speed is used. In Figure 6 on the next page the rotor speed variation due to a wind speed step is compared between HAWC2 and HAWCStab2 at 15, 20, and $25 \mathrm{~m} / \mathrm{s}$. The gain-scheduling Lin. and Quad.+Damp. are shown. The time response of the two models appears to be of good agreement. The maximum amplitude of the overspeed, the time at which the maximum overspeed occurs and the time the system needs to reach the steady state give an indication that the two models agree on the values of the regulator mode frequency and damping. When using the gain-scheduling Lin. the system appears to be slower at regaining the steady state speed for increasing wind speed, indicating an increase of the damping.

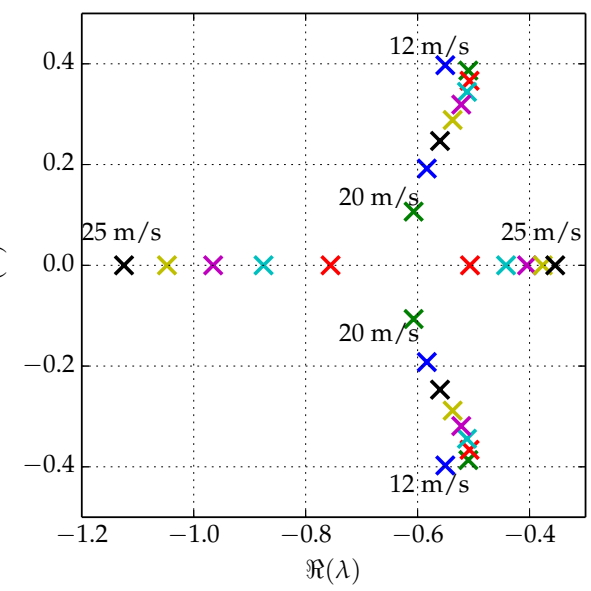

Figure 5. Trajectory of the pole associated with the speed regulator mode for increasing wind speed. Poles obtained with a linear fitting of the aerodynamic gain and without aerodynamic damping (Lin.). 


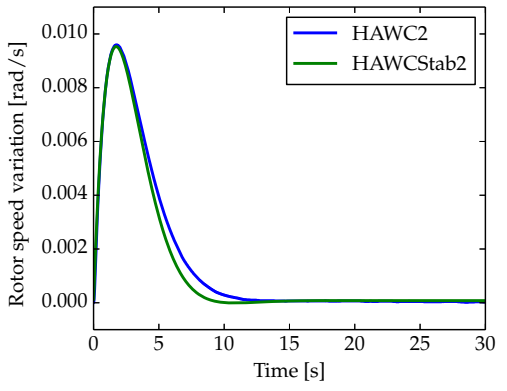

a) Lin. at $15 \mathrm{~m} / \mathrm{s}$

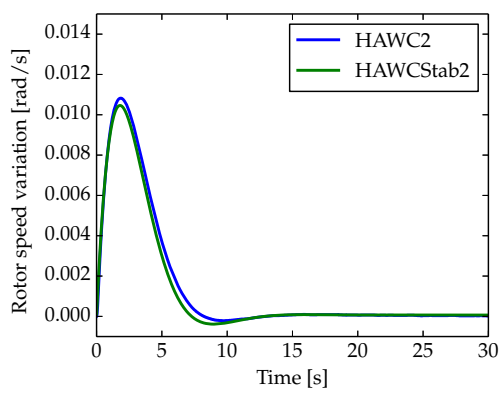

d) Quad.+Damp. at $15 \mathrm{~m} / \mathrm{s}$

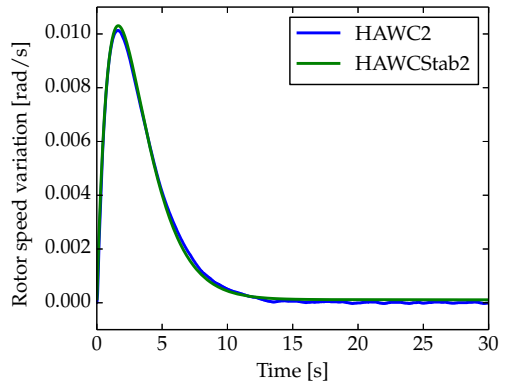

b) Lin. at $20 \mathrm{~m} / \mathrm{s}$



e) Quad.+Damp. at $20 \mathrm{~m} / \mathrm{s}$

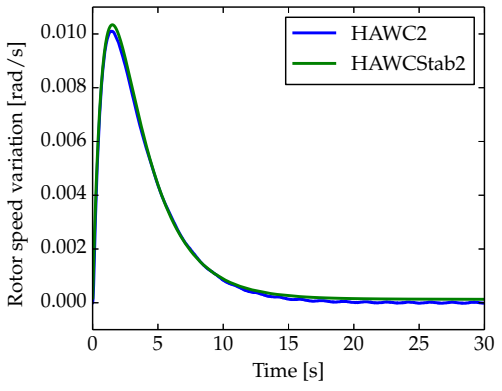

c) Lin. at $25 \mathrm{~m} / \mathrm{s}$

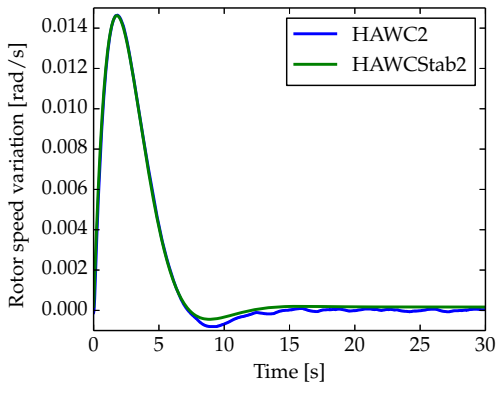

f) Quad.+Damp. at $25 \mathrm{~m} / \mathrm{s}$

Figure 6. Rotor speed variation due to a wind speed step. Comparison between HAWC2 and HAWCStab2 at 15,20 and $25 \mathrm{~m} / \mathrm{s}$. Linear and quadratic gain-scheduling with additional damping term. Rigid wind turbine and steady aerodynamic.

On the other hand when using the Quad.+Damp. gain-scheduling, the rotor speed has similar response at the different wind speeds. The overspeed value is different because the sensitivity of the aerodynamic torque to a wind speed variation changes with the wind speeds. The shape of the response is simply scaled by a factor indicating similar frequency and damping of the regulated mode.

\section{B. High-order model}

In this section the performances of the gain-scheduling methods are compared closing the loop with a highorder model. The model is obtained with HAWCStab2. The model includes a fully flexible turbine, unsteady aerodynamics and a second-order filter of the rotor speed for the PI controller (natural frequency of $0.6 \mathrm{~Hz}$ and damping ratio of 0.7 ). The controller tuning is performed to assign to the regulated pole a frequency of $0.1 \mathrm{~Hz}$ and a damping ratio of 0.7 , Figure 3 on page 5 . Figure 7 on the next page shows the damped natural frequency and the damping ratio of the rotor speed regulator mode for the high-order model. It appears immediately that all the three approaches fail at placing the pole where it is asked with the tuning. The damped natural frequency results at higher values while the damping at lowers. When using only the aerodynamic gain in the gain-scheduling (Lin. and Quad.) the natural frequency grows linearly with the wind speed. The addition of the aerodynamic damping, Quad.+Damp. improves the location of the pole reducing the dependency on the wind speed and reducing the minimum value. The opposite happens for the damping. The Lin. and Quad. approaches have a lower damping in the whole wind speed range compared to the more complex method. The Quad.+Damp. approach seems more capable at pulling up, closer to the reference value, the damping for increasing wind speeds. For the full model the situation is critical at the beginning of the operational region. Here indeed the damping ratio drops considerably approaching $0 \%$ instead of the desired $70 \%$. The more detailed gain-scheduling approach Quad.+Damp. is able to increase the damping compared to the simple one Lin. but, still, the value of the damping is significant lower than the desired one. At low wind speeds the scheme Quad. achieves the same performances of the more complex method Quad.+Damp.. The reason of this poor performance of all the approaches is partially due to the presence of the second-order rotor speed filter. Figure 8 on the following page shows the effect of the secondorder filter on the damped frequency and the damping ratio of the rotor speed regulator mode. When 


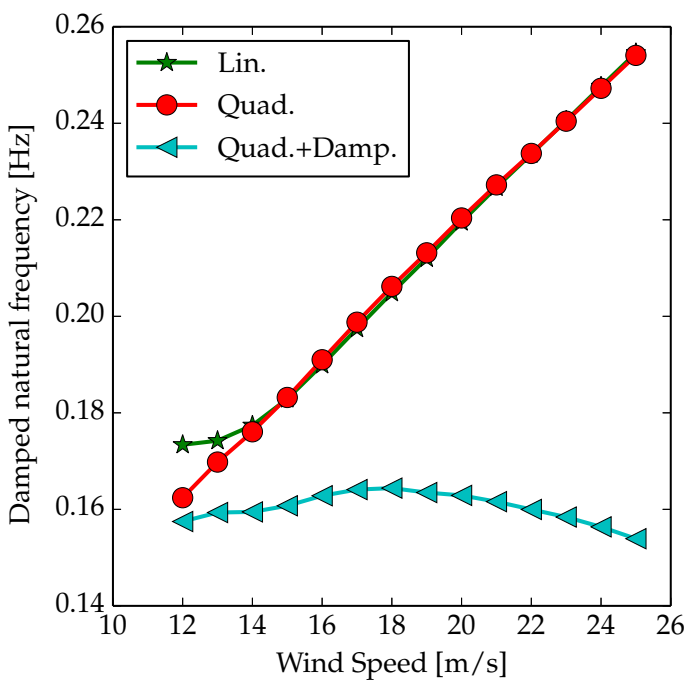

a) Damped natural frequency.

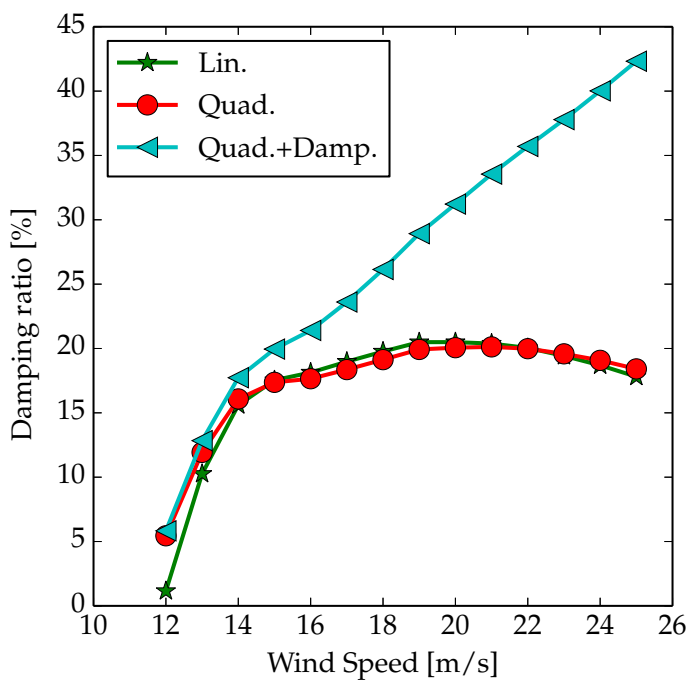

b) Damping ratio.

Figure 7. Damped natural frequency and damping ratio of the rotor speed regulator mode using a high-order model of the wind turbine. Comparison of the three gain-scheduling schemes.

removing the filter the damped natural frequency of the regulator mode decreases in all the range except at $12 \mathrm{~m} / \mathrm{s}$. The maximum difference occurs in the central part of the operational region when a reduction of approximately $6 \%$ occurs. The damping ratio shows significant differences when removing the filter. The minimum value goes from approximately $5 \%$ to $30 \%$. Since it is not possible to remove the rotor speed filter for real application, because the drivetrain mode would be excited by the pitch action, a significant care must be taken when deciding its cut-off frequency.

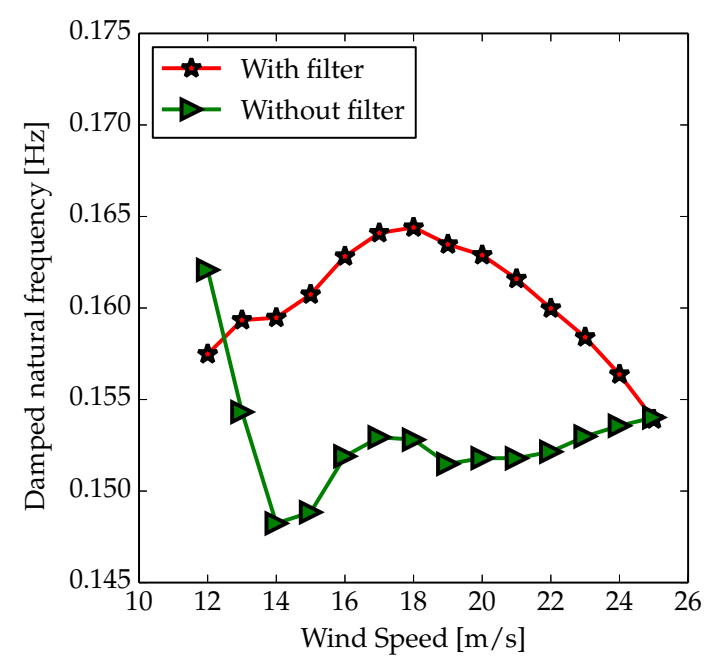

a) Damped natural frequency.

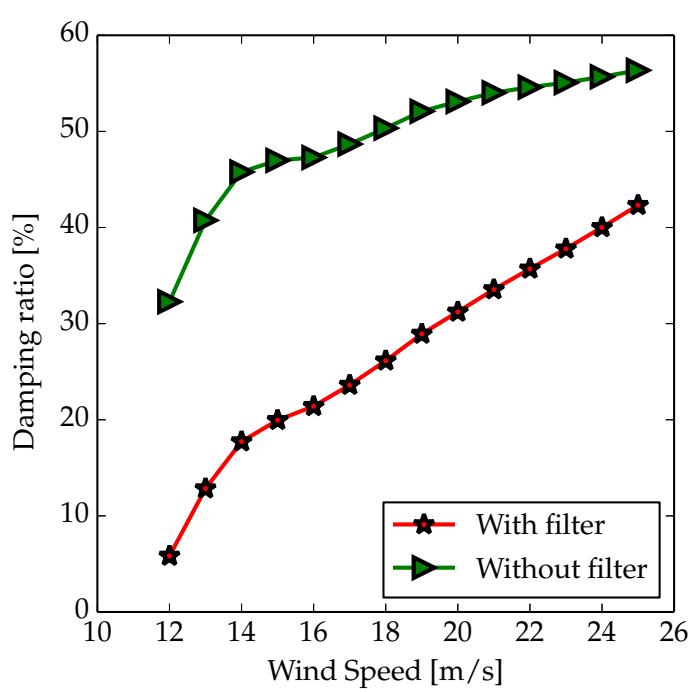

b) Damping ratio.

Figure 8. Damped natural frequency and the damping ratio of the rotor speed regulator mode using a high-order model of the wind turbine. Comparison of the influence of the second-order rotor speed filter when using the gain-scheduling that includes the aerodynamic damping Quad.+Damp.. 


\section{Comparison of the linear and non-linear model}

In this section the systems obtained with the high-order linearized model are compared with results computed with a non-linear model obtained with HAWC2. Figure 9 shows the comparison between HAWCStab2 and HAWC2 results of the rotor speed response to a wind speed step. The wind speeds are 15,20 , and $25 \mathrm{~m} / \mathrm{s}$. Only the Lin. and Quad.+Lin. gain-scheduling methods are compared. The comparison shows a similar behavior in both models. In the time responses it is possible to identify three different modes that are excited by the wind speed steps the regulator mode, the drivetrain mode, and the first lateral tower mode. The regulator mode is the dominant one. The drivetrain mode can be noticed close to the maximum overspeed as a high frequency variation. The first lateral tower mode can be noticed once the regulator mode oscillations are damped out. The frequency and the damping of the regulator mode are in a satisfactory agreement between the two models. For both gain-scheduling methods the damping increases for increasing wind speed, as shown in the previous section.

\section{Effects on the wind turbine aeroservoelastic modes}

Figure 10 on the following page shows the effect of the gain-scheduling method on the closed-loop aeroservoelastic wind turbine modes. The only modal damped natural frequency that is affected is the first longitudinal tower mode. When using the Quad.+Damp. method the frequency of the first longitudinal tower mode decreases for increasing wind speeds. At $25 \mathrm{~m} / \mathrm{s}$ the first longitudinal tower frequency is almost coinciding with the first lateral tower mode. When looking at the first modes it also appears that when the aerodynamic gain is neglected, the speed regulator damped frequency increases with the wind speed and it approaches the first tower modes. This reduction in the frequency gap could be a problem when the tower has a lower frequency or when a more aggressive speed regulation (i.e. higher natural frequency of the speed regulator mode) is required. In these cases the two modes might coincide and high loads due to tower excitation could occur. Hence, taking into account also the aerodynamic damping in the gain-scheduling reduces the risk of running into tower excitation problems. Figure 11 on the next page shows the effect of the gain-scheduling

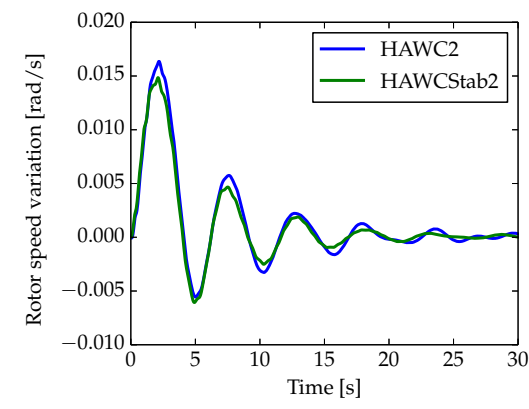

a) Lin. at $15 \mathrm{~m} / \mathrm{s}$

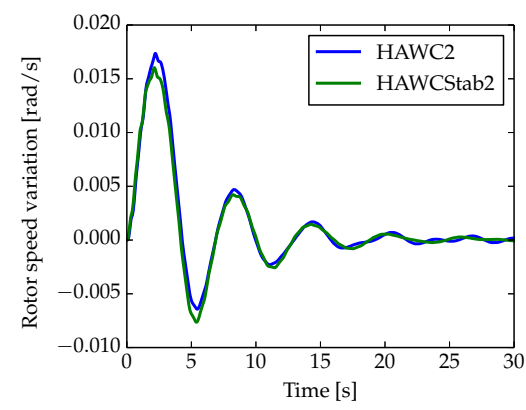

d) Quad.+Damp. at $15 \mathrm{~m} / \mathrm{s}$

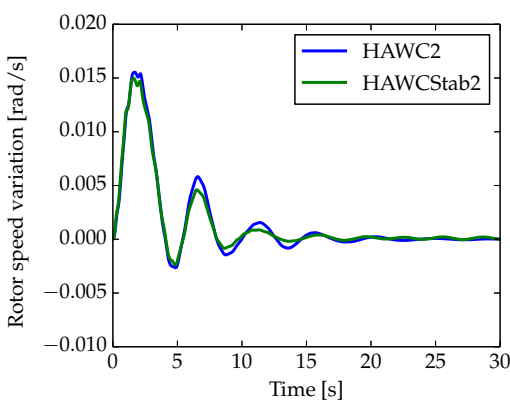

b) Lin. at $20 \mathrm{~m} / \mathrm{s}$

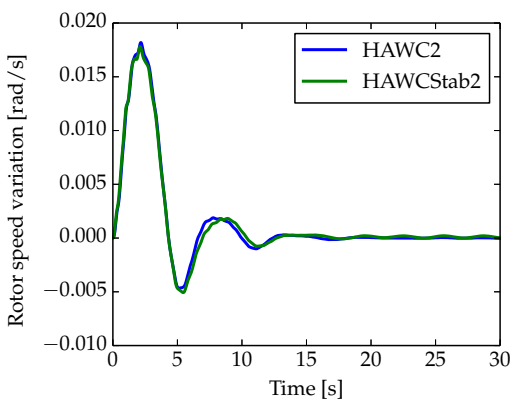

e) Quad.+Damp. at $20 \mathrm{~m} / \mathrm{s}$

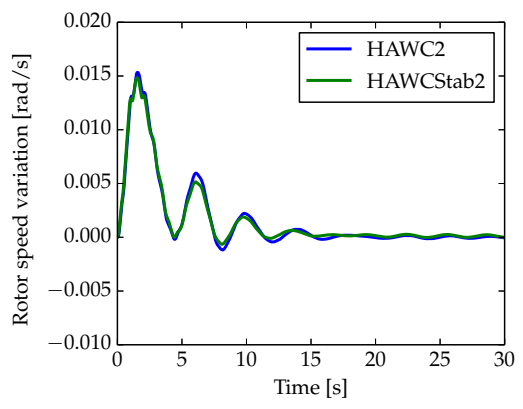

c) Lin. at $25 \mathrm{~m} / \mathrm{s}$

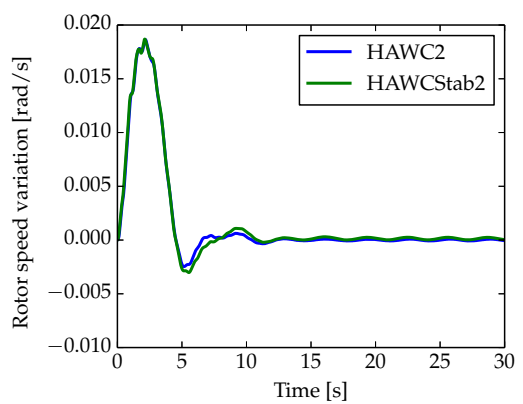

f) Quad.+Damp. at $25 \mathrm{~m} / \mathrm{s}$

Figure 9. Rotor speed response to a wind speed step. Comparison between HAWC2 and HAWCStab2 at 15, 20 and $25 \mathrm{~m} / \mathrm{s}$. Linear and quadratic gain-scheduling with additional damping term. Fully flexible wind turbine and unsteady aerodynamic 


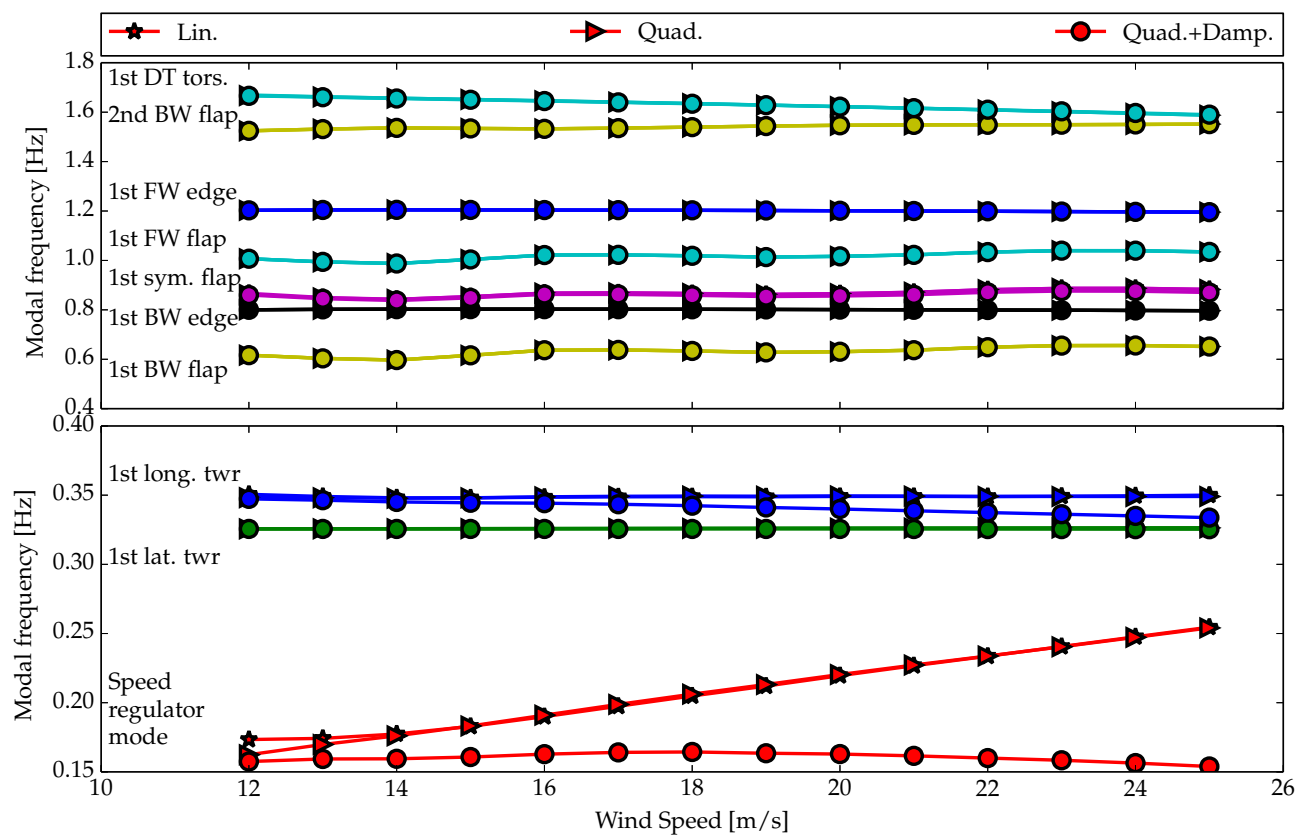

Figure 10. Closed loop aeroservoelastic damped natural frequencies of the first 10 turbine modes. Comparison between the gain-scheduling methods.

methods on the damping ratio of the first ten closed-loop aeroservoelastic wind turbine modes. Other than the speed regulator mode the first longitudinal tower mode appears to be affected by the different gainscheduling schemes. As for the frequency, when using the method Quad.+Damp. the damping decreases with increasing wind speed, going from $15 \%$ to $10 \%$. The first drivetrain mode is also affected. At low wind speeds the damping ratio is slightly higher when using Quad.+Damp. compared to Lin.. This increase can

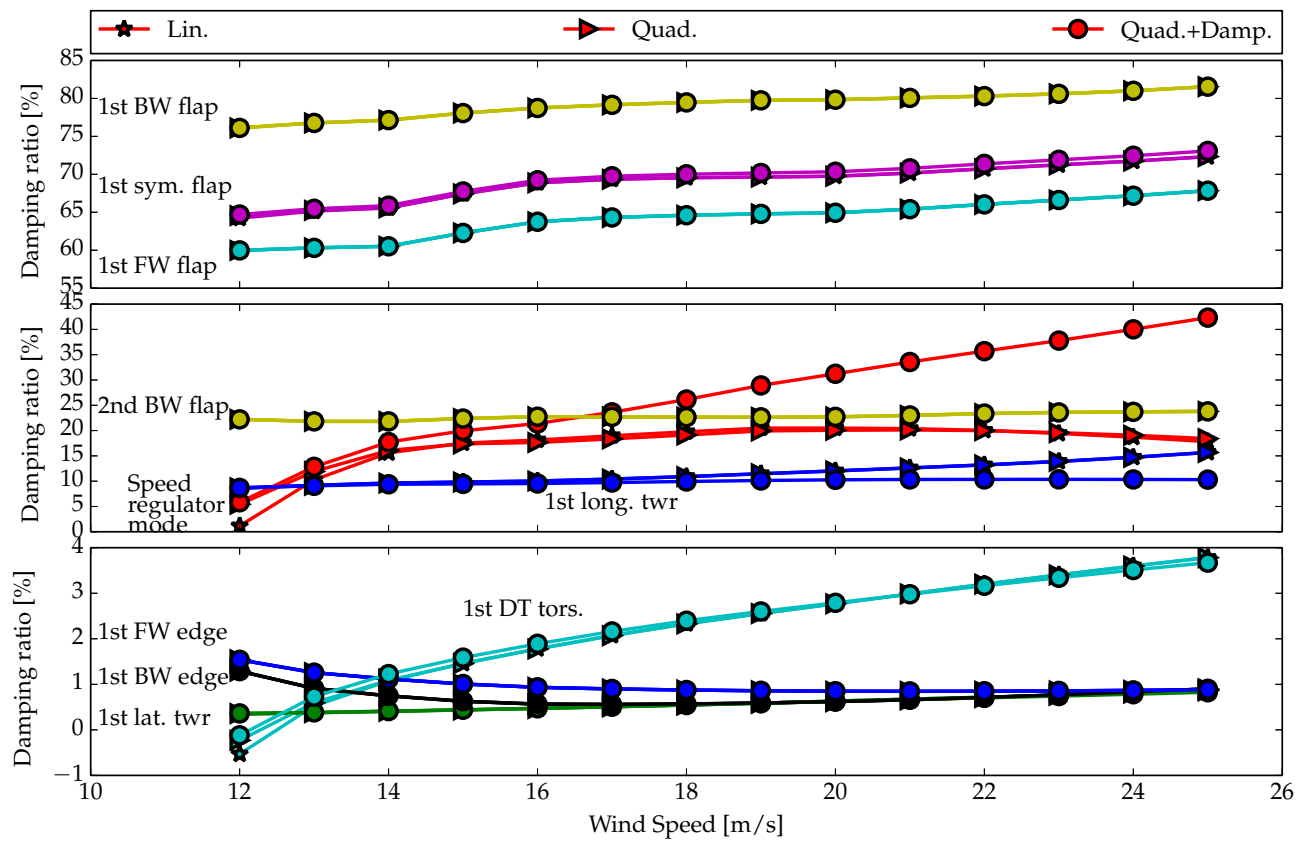

Figure 11. Closed loop aeroservoelastic damping ratios of the first 10 wind turbine modes. Comparison between the gain-scheduling methods. 
be important since the mode is low damped close to the switching between the operational regions. In the case under evaluation the mode even results negatively damped. None of the other first ten wind turbine modes seems to be noticeably affected by the choice of the gain-scheduling method.

\section{Effects on the loads}

In this section the gain-scheduling schemes are compared looking at their effects on wind turbine loads. The loads are computed with turbulent wind simulations performed with HAWC2. Three different wind speeds are analyzed 12,15 , and $25 \mathrm{~m} / \mathrm{s}$. The turbulence intensity at each wind speed is set according to the wind turbine class II-B. ${ }^{12}$ Figure 12 shows the variation with respect to the Lin. scheme of the standard deviation of the rotor speed and tower base longitudinal bending moment. The rotational speed has a higher standard deviation for both methods (Quad. and Quad.+Damp.) at all the wind speeds. The reason for this increase is due to the different location of the regulator mode. Since a lower frequency of the regulator mode means that the controller action is less aggressive, higher rotor speed variations are expected. The Quad. method has a lower frequency compared to Lin. at low wind speeds, see Figure 10 on the previous page, and that justifies the $5 \%$ increases in the rotor speed standard deviation seen in Figure 12 a). For higher wind speeds the difference in the frequency is minimal and therefore no significant variation in the standard deviation is present. With the Quad.+Damp. scheme the regulator frequency is always lower compared to the method Lin. and the gap increases with the wind speed. Hence, the increasing standard deviation for increasing wind speed when using the Quad.+Damp. method. A controller with a lower regulator frequency is more likely to generate less loads on the tower since less thrust variations are present and there is less tower excitation due to the higher gap between the controller frequency and the tower frequency. The lower load can be seen in Figure 12 b) where the standard deviation of the tower base longitudinal moment decreases when using the Quad.+Damp. scheme compared to the Lin. and Quad..

\section{Conclusions}

A comparison of different gain-scheduling schemes for a pitch regulated wind turbine PI controller has been performed. A new gain-scheduling has been derived and introduced. The new approach also takes into account the dependency of the aerodynamic torque on the rotational speed. When using a high-order linear model the placement of the regulator mode is modified by the other wind turbine modes. The damping of the regulator decreases and it gets close to negative values at to the beginning of the above-rated region. The regulator mode frequency is shifted at higher values compared to the designated one. The new gainscheduling improves the placement of the regulator pole guaranteeing an almost constant mode frequency and a higher damping compared to the traditional methods. The lower regulator frequency leads to a less

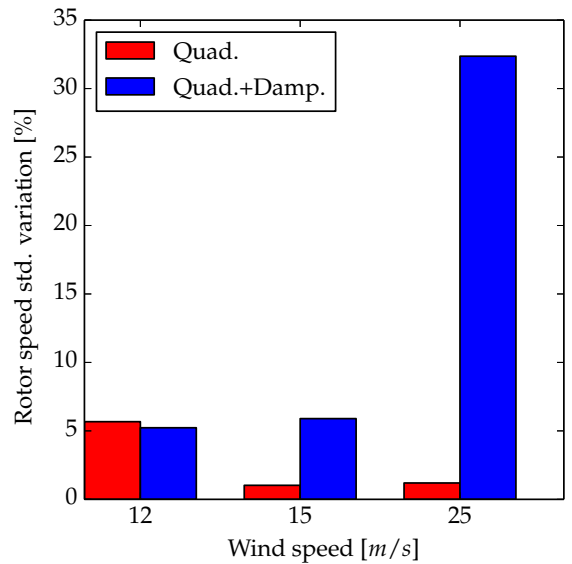

a) Rotor speed

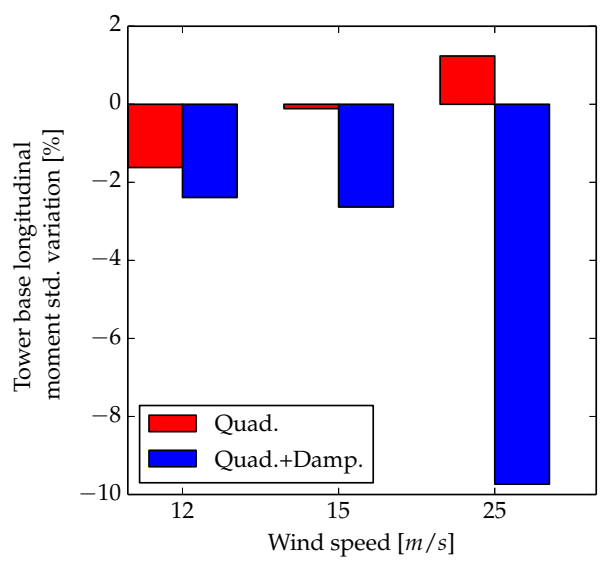

b) Tower base long. moment

Figure 12. Percentage increase with respect to the Lin. case of the standard deviation of the rotor speed and tower base longitudinal bending moment. 
aggressive rotor speed regulation and hence to higher rotor speed variations. On the other hand it appears that the standard deviation of the loads at the tower base is reduced.

\section{References}

${ }^{1}$ Hansen, M. H., Hansen, A., Larsen, T. J., Øye, S., Sørensen, P., and Fuglsang, P., "Control design for a pitch-regulated, variable speed wind turbine," Tech. Rep. Ris $\varnothing-$ R-1500(EN), Ris $\varnothing$ National Laboratory, Roskilde, Denmark, 2005.

${ }^{2}$ Wright, A. D. and Fingersh, L. J., "Advanced Control Design for Wind Turbines; Part I: Control Design, Implementation, and Initial Tests." Research Report TP-500-42437, NREL, 2008.

${ }^{3}$ Bossanyi, E. A., "The Design of closed loop controllers for wind turbines," Wind Energy, Vol. 3, No. 3, 2000, pp. 149-163.

${ }^{4}$ Hansen, M. H., "Aeroelastic Optimization of MW Wind Turbines," Tech. Rep. Ris $\varnothing-R-1803(E N)$, Ris $\varnothing$ National Laboratory, Denmark, 2011.

${ }^{5}$ Riziotis, V. A., Politis, E. S., Voutsinas, S. G., and Chaviaropoulos, P. K., "Stability analysis of pitch-regulated, variablespeed wind turbines in closed loop operation using a linear eigenvalue approach," Wind Energy, Vol. 11, No. 5, 2008 , pp. 517-535.

${ }^{6}$ Larsen, T. J. and Hansen, M. A., "How 2 HAWC2, the user's manual," Tech. Rep. Ris $\emptyset-$ R-1597(ver. 3-1)(EN), Ris $\varnothing$ National Laboratory, Denmark, 2007.

${ }^{7}$ Jonkman, J., Butterfield, S., Musial, W., and Scott, G., "Definition of a 5-MW Reference Wind Turbine for Offshore System Development," Tech. Rep. NREL/TP-500-38060, NREL/NWTC, 2009.

${ }^{8}$ Hansen, M. H., "Aeroelastic stability analysis of wind turbines using an eigenvalue approach," Wind Energy, Vol. 7 , No. 2, 2004, pp. 133-143.

${ }^{9}$ Hansen, M. H., "Aeroelastic Properties of Backward Swept Blades," American Institute of Aeronautics and Astronautics, Orlando, Florida, 2011, p. 119.

${ }^{10}$ Sønderby, I. and Hansen, M. H., "Open-loop frequency response analysis of a wind turbine using a high-order linear aeroelastic model," Wind Energy, 2013, pp. n/an/a.

${ }^{11}$ Hansen, M. H. and Henriksen, L. C., "Basic DTU Wind Energy controller," Tech. Rep. E-0028, DTU Wind Energy, 2013.

${ }^{12}$ IEC/TC88, IEC 61400-1 Ed.3: Wind turbines - Part 1: Design requirements, 2005. 